Brit. Heart f., 1966, 28, 359.

\title{
Carbon Monoxide Diffusion of Lungs in Assessment of Pulmonary Blood Flow in Patients with Intracardiac Shunts
}

\author{
C. M. LEWIS AND A. J. BRINK \\ With the technical assistance of $M$. J. THERON
} From the Department of Internal Medicine and the Degenerative Diseases Group of the Council for Scientific
and Industrial Research, University of Stellenbosch Medical School, Bellville, Cape Province, South Africa

There are several techniques that may be used to measure the pulmonary blood flow. We wished to determine whether a relatively simple test, entailing minimal interference with the patient, could be employed to give a reasonably accurate assessment of the size of intracardiac shunts. Standard methods currently employed in the determination of pulmonary blood flow in cases of intracardiac shunts include cardiac catheterization studies with application of the Fick principle, and various indicator-dilution techniques. None of these procedures is, however, within the scope of ordinary clinical examination, and alternative methods have been sought.

Use has, for instance, been made of the observation that the increased pulmonary blood flow associated with most cases of atrial septal defect may effect changes in the appearance of the pulmonary vasculature, as viewed in the routine 6-foot chest radiograph made with held respiration in the erect posture (Healey et al., 1949; Campbell, 1951; Kraemer et al., 1955; Fouché, Beck and Schrire, 1963). Kraemer et al. (1955) reported that there was no apparent quantitative relation between physiological and radiographic changes in atrial septal defect, as judged by the parameters of pulmonary flow index on one hand and the degree of lung field vascularity on the other. Fouché et al. (1963), however, found that a correlation did exist between percentage left-to-right shunt and the degree of pulmonary plethora in cases of secundumtype atrial septal defect, when the normal gradation of vascular markings between apex and base was taken into account. A rough correlation was also

Received April 12, 1965. found to exist between cardiothoracic ratio and percentage left-to-right shunt.

Bedell (1961) undertook studies in connexion with the diffusing capacity for carbon monoxide in the lungs of patients with atrial and ventricular defects. This work was subsequently supplemented (Bedell and Adams, 1962) with studies in normal controls, at rest and under conditions of exercise. It was found that the single-breath carbon monoxide diffusion technique yielded significantly higher values for diffusing capacity $\left(\mathrm{DL}_{\mathrm{co}}\right)$ in cases of intracardiac defect than were found in normal subjects; and that the values for $\mathrm{DL}_{\mathrm{co}}$ were higher for atrial septal defects than for ventricular defects. There was also a tendency towards higher $\mathrm{DL}_{\mathrm{CO}}$ values with increase in pulmonary flow.

In the present study, the simple Milledge (1960) technique for determining $\mathrm{CO}$ diffusion was used in a series of tests carried out in 16 patients with intracardiac shunts, so that the findings could be related to the radiological appearance of the lung fields and the hæmodynamic status (Table). In a separate control series of 17 subjects with no known increase in pulmonary blood flow or underlying cardiovascular disease carbon monoxide diffusion was determined by the same technique and the radiological appearances evaluated.

\section{METHODS}

For measuring $\mathrm{CO}$ diffusion we made use of the Milledge (1960) "steady state" technique, as modified by Wassermann (1961), which employs a pallado-sulphite detector in the estimation of carbon monoxide (CO) concentration. By means of this technique we were 359 
TABLE

HÆMODYNAMIC AND DIFFUSION PARAMETERS IN 16 PATIENTS WITH CARDIAC SHUNTS

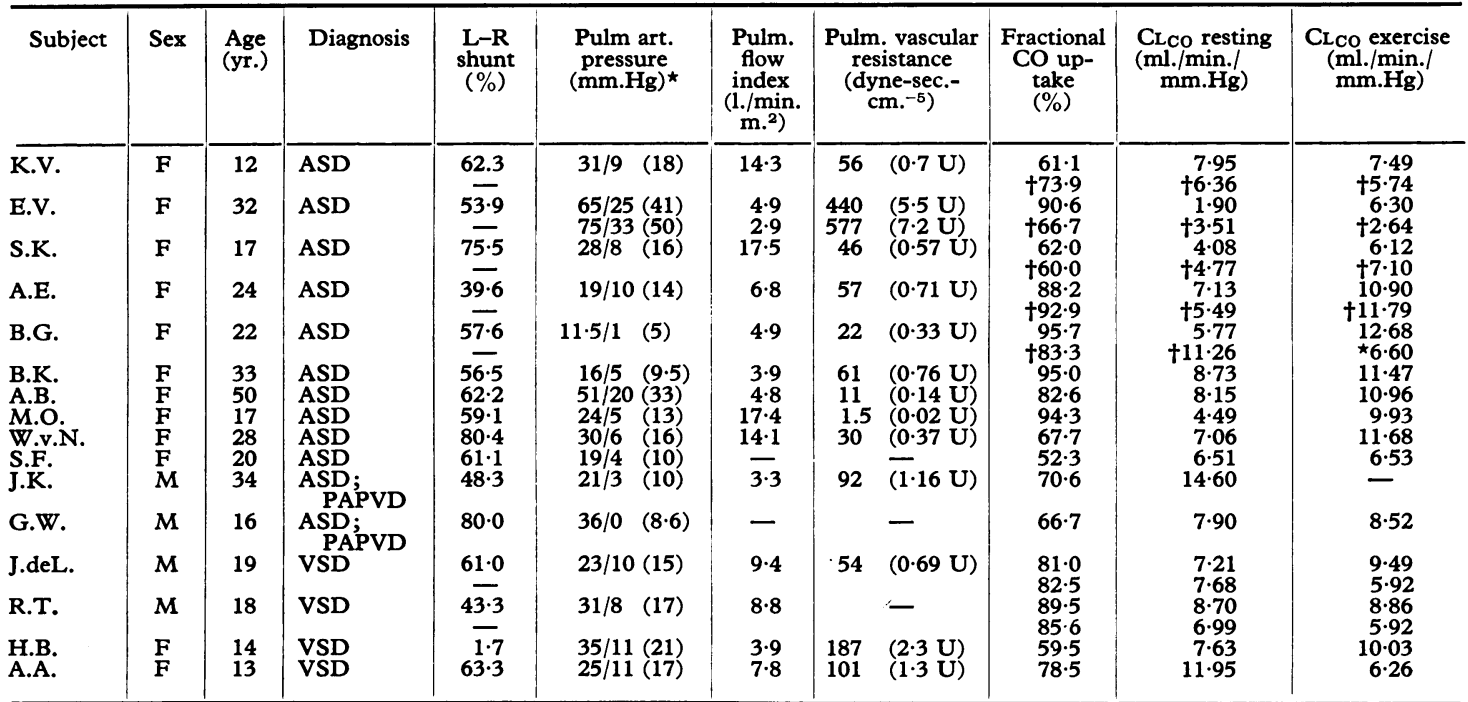

ASD, atrial septal defect; VSD, ventricular septal defect; PAPVD, partial anomalous pulmonary venous drainage.

* Mean pulmonary artery pressure in parenthesis.

† Post-operative determination.

"conductance" $\left(\mathrm{CL}_{\mathrm{CO}}\right)$ under resting conditions. The use of these two indices avoids the necessity for obtaining a representative end-tidal sample of expiratory gas as required in the assessment of diffusing capacity $\left(\mathrm{DL}_{\mathrm{Co}}\right)$, the partial pressure of $\mathrm{CO}$ in the inspiratory gas mixture alone requiring to be known. The term "conductance" was first proposed by Dornhorst (1952). It has since been employed by MacNamara, Prime, and Sinclair (1959) at the Brompton Hospital in studies of CO diffusion. This determination differs from the more customary index of diffusing capacity only in that the $\mathbf{P}_{\mathrm{Co}}$ of the inspiratory gas rather than that of an end-tidal sample is used in the calculation. MacNamara has made the observation that while $\mathrm{CL}_{\mathrm{co}}$ will not be of assistance in drawing a sharp distinction between patients and normal persons, it will in fact provide an accurate measure of the efficiency of the gas-conducting pathways taken as a whole. On the other hand, he concluded that $\mathrm{CO}$ percentage uptake was as useful as $\mathrm{DL}_{\mathrm{CO}}$ in differentiating normal and abnormal lungs. Variations in the degree of CO uptake are determined chiefly by changes in the alveolar surface area available for diffusion. In the absence of highly irregular alveolar ventilation, therefore, this simple test appears to be of value in determining pulmonary alveolar diffusing capacity.

The radiological assessment of the degree of pulmonary blood flow in intracardiac shunts was effected by means of a "double-blind" procedure. Routine 6-foot radiographs of the chest were studied in patients who had previously undergone hæmodynamic and diffusion studies, and also in a control series of subjects known to have normal pulmonary blood flow. In each case the cardiac shadow and pulmonary hilar vessels were screened from view, so that the radiologist undertaking the assessment would not be influenced by the size and shape of the heart and major vessels in his estimate of pulmonary vascularity. Independent opinions were received from 3 radiologists. Final conclusions on the assessment of pulmonary blood flow were expressed simply as: increased, diminished, or normal, without further attempt at quantitation (Fig. 3).

\section{Results}

The mean normal value for CO percentage uptake was $55.0 \pm 15 \cdot 06$, and that for intracardiac shunts was $77 \cdot 3 \pm 14 \cdot 34$ (Fig. 1A). The difference between these two values was highly significant $(p<0.001)$. There was, however, no significant difference between the mean $\mathrm{CO}$ percentage uptake in the small group of ventricular septal defects and the mean value determined in the atrial septal defects. The large standard deviations may be ascribed to the measure of subjectivity attaching to the technique for determining carbon monoxide concentrations in the respiratory gas mixture. Our mean value for normal subjects is similar to the average previously determined by Wassermann (1961) in this laboratory $(52.5 \%)$ and to those values determined by Milledge (1960) using the same technique. Milledge found an average value of 52 per cent in his series of 14 healthy subjects, individual values ranging from 43 to 65 per cent. MacNamara et al. (1959) recorded a mean value of $51 \pm 4.66$ per cent, and Bates' (1952) figure was $53 \cdot 0 \pm 2 \cdot 24$ per cent. 


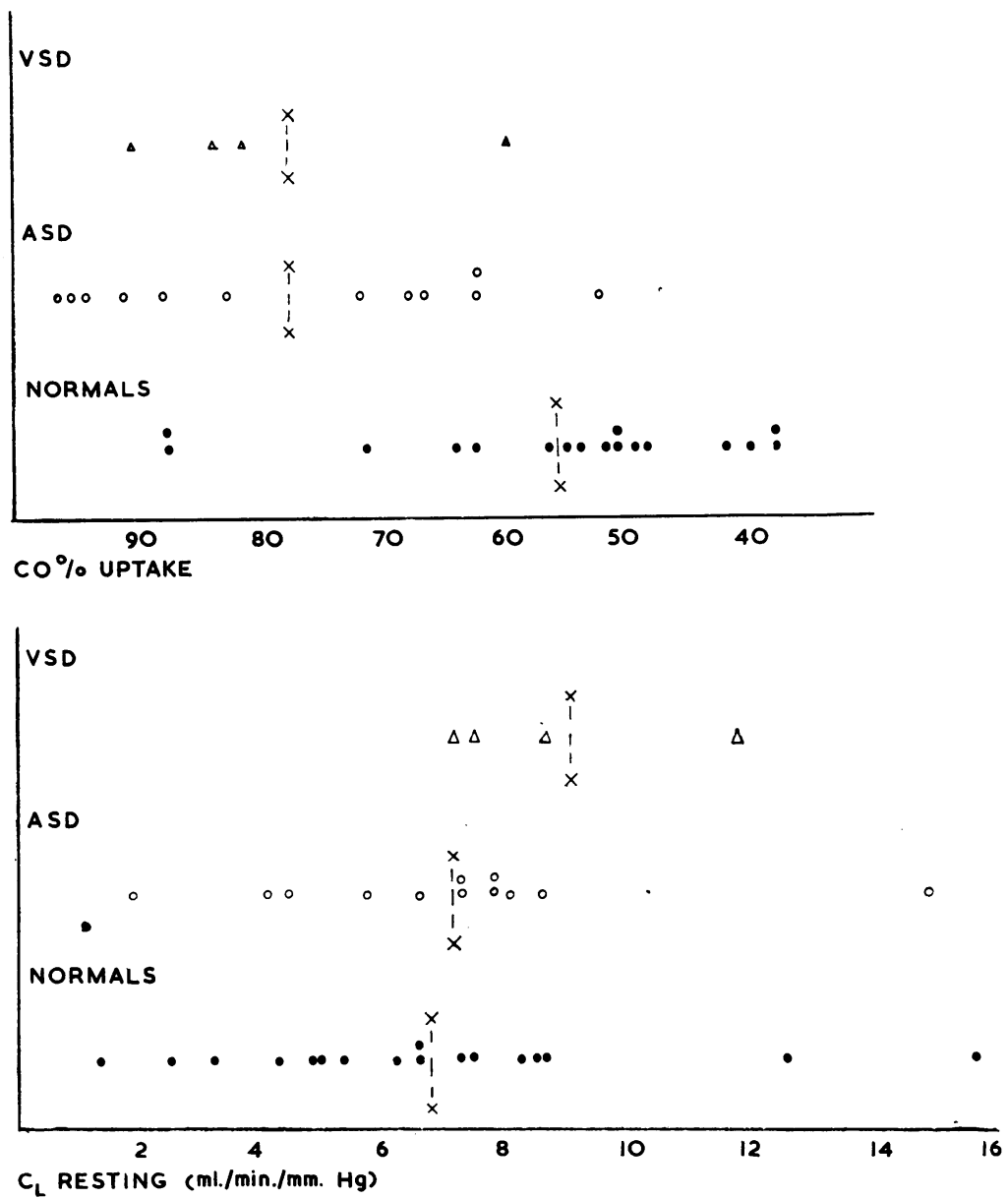

FIG. 1A.-Determinations of fractional carbon monoxide uptake in a group of 16 patients with interatrial or interventricular septal defects and in a group of 17 normal subjects.

FIG. 1B.-Determinations of carbon monoxide "conductance" under resting conditions in 16 patients with intracardiac septal defects and in a group of 17 normal subjects.

The mean values for $\mathrm{CL}_{\mathrm{Co}}$ in normal subjects and in patients with intracardiac shunts were respectively: $6.69 \pm 3.55$ and $7.49 \pm 2.95 \mathrm{ml} . / \mathrm{min} . / \mathrm{mm} . \mathrm{Hg}$ (Fig. 1B). The difference between the two means was not significant. Both values fell within the limits of normal established in this laboratory by Wassermann (average 8.54) and compare favourably with MacNamara's mean of $6.5 \pm 1.66$. A normal increase in $\mathrm{CL}_{\mathrm{CO}}$ with exercise was found in both control subjects and patients.

Both diffusion parameters, fractional CO uptake, and $\mathrm{CL}_{\mathrm{co}}$, were correlated with the hæmodynamic parameters of percentage left-to-right shunt (i.e. the percentage quotient of pulmonary flow less systemic flow divided by pulmonary flow) and the pulmonary index expressed in litres/min. m. ${ }^{2}$ (Fig. 2A-2D). In the case of $\mathrm{CL}_{\mathrm{Co}}$ no correlation could be shown to exist with either percentage shunt (Fig. 2C) or pulmonary index (Fig. 2D). When $\mathrm{CO}$ percentage uptake was correlated with pulmonary index a negative correlation was demonstrated $(r=-0.55)$ (Fig. 2B). There was, however, no correlation between $\mathrm{CO}$ uptake and percentage shunt (Fig. 2A).

In some cases, follow-up studies were performed after surgical repair of the intracardiac defect, and with a single exception no substantial changes in either fractional CO uptake or conductance were demonstrable (Table). The follow-up period was, however, no longer than 10 weeks in the majority of cases.

A significant degree of pulmonary arterial hypertension was present in only one of the cases of atrial septal defect studied (Subject E.V., Table). The 

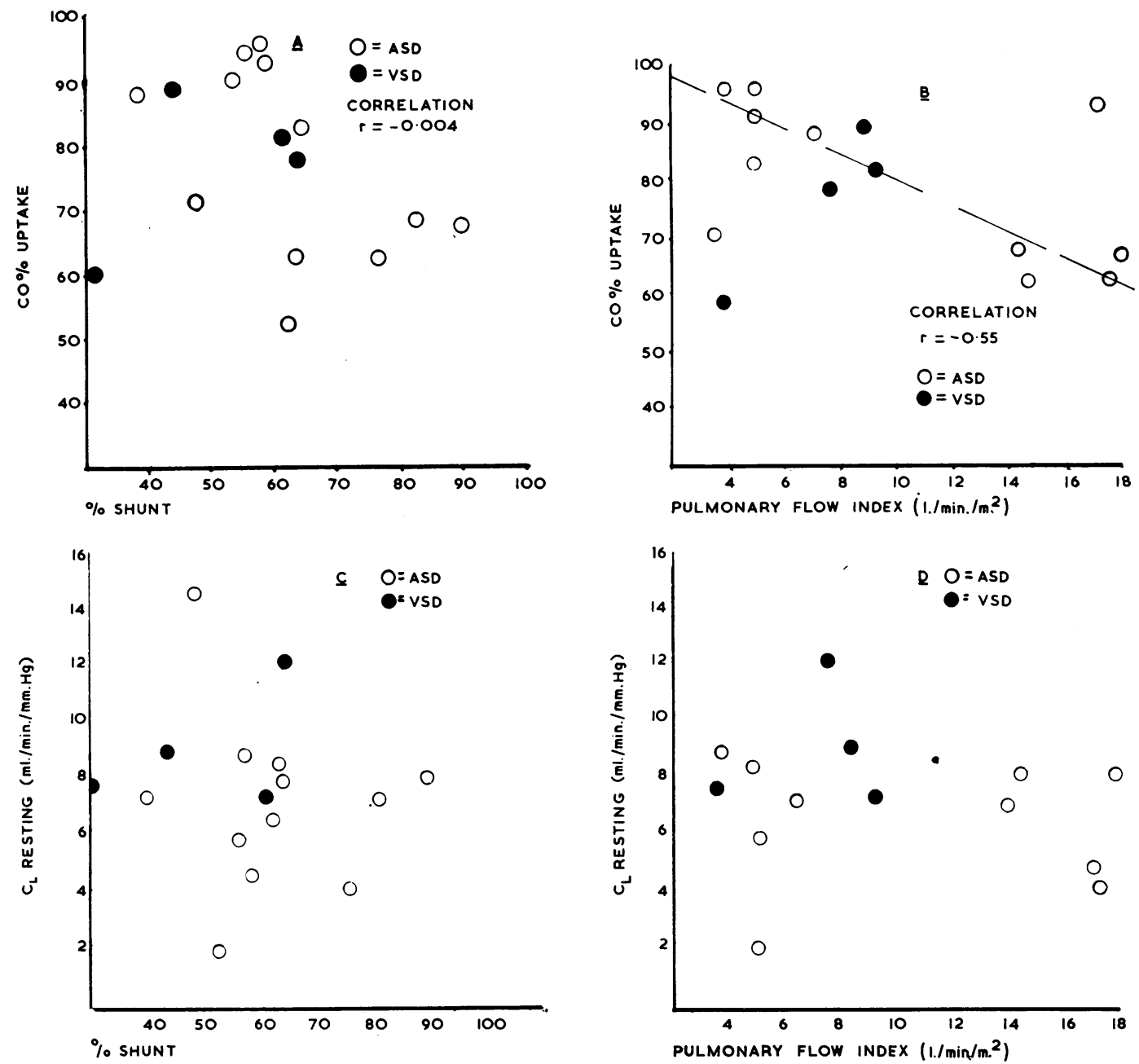

FIG. 2A.-The results of graphic plotting of fractional carbon monoxide uptake against the percentage left-to-right shunt in group of patients with intracardiac septal defects. The coefficient of correlation ( $r$ ) was +0.004 .

FIG. 2B.-Graphic plotting of fractional carbon monoxide uptake against pulmonary index. A significant negative correlation is demonstrated $(r=-0.55)$.

FIG. 2C.-Graphic plotting of resting carbon monoxide conductance against percentage left-to-right shunt showing the absence of correlation between these two parameters.

FIG. 2D. - The relation between resting carbon monoxide conductance and pulmonary index. No correlation exists between these parameters.

pulmonary arteriolar resistance was also abnormally high in this patient. After closure of the septal defect the degree of pulmonary hypertension increased progressively. One year after surgery the pulmonary vascular resistance had increased from 5.5 to 7.2 units. Concomitantly there was a substantial increase in the grade of exertional dyspnoea. During the same period the CO percentage uptake declined from $90 \cdot 6$ to $66 \cdot 7$. The
$\mathrm{CI}_{\mathrm{co}}$ was abnormally low, both before and after operation.

In the radiological assessment of pulmonary blood flow the following results were obtained (Fig. 3). Of the normal subjects, 51 were regarded as having normal pulmonary blood flow, 4 were regarded as plethoric, and one as having an oligæmic appearance. Of the patients with atrial septal defects, 20 were reported as having increased 
pulmonary flow, and a further 13 had a normal flow. In no instance was the flow regarded as diminished. Opinions expressed in respect of the patients with ventricular septal defect were that 7 had a plethoric appearance, 10 appeared normal, and one was oligæmic.

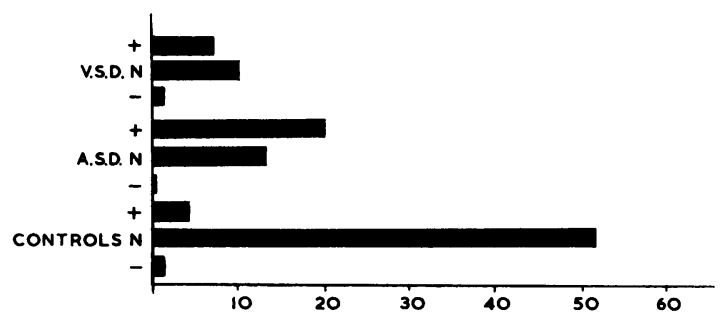

Fig. 3.-The results of independent assessments by 3 radiologists of the chest radiographs of a group of patients with intracardiac shunts and a control series of normal subjects in respect of radiographic appearances of pulmonary vascularity. Assessments are expressed in terms of normal vascularity $(\mathbf{N})$, plethora $(+)$, or oligæmia $(-)$, and represent subjective opinions in a blind trial procedure.

\section{Discussion}

Our finding of a significantly higher fractional CO uptake in patients with intracardiac shunts is in agreement with the observations of a number of workers (Rankin and Callies, 1958; Rankin, 1960; Bedell, 1961; Bedel and Adams, 1962). We were, however, unable to confirm the finding of Bedell (1961) that there is a relatively lower CO percentage uptake in ventricular septal defect. Our series of ventricular septal defects was, however, very small.

As MacNamara et al. (1959) has suggested, it seems that conductance $\left(\mathrm{CL}_{\mathrm{co}}\right)$ cannot usefully be employed to distinguish subjects with septal defects from normal subjects. In only one instance was a very low resting $\mathrm{CL}_{\mathrm{co}}$ observed, namely in the patient E.V. (Table) who had a significantly raised pulmonary vascular resistance, and who may be presumed to have had structural changes in the pulmonary capillary bed. An over-all deficiency in gas transfer as a result of such changes seems a likely explanation for the lowered resting $\mathrm{Cl}_{\mathrm{CO}}$. If this is true, then the progress of pulmonary arterial hypertension and an increase in vascular resistance would parallel a decline in diffusing capacity. Evidence in support of this has been forthcoming from Rankin (1960). He has shown that in cases of mitral stenosis, the early increase in $\mathrm{DL}_{\mathrm{co}}$ seen in the stage of capillary congestion and a relatively normal membrane has been succeeded by decreased diffusing capacity on the advent of pulmonary arterial hypertension and membrane thickening.

The general good correlation between radiological assessment of pulmonary flow and direct hæmo- dynamic determinations on the one hand, and the established increase in diffusion associated with conditions of enhanced pulmonary flow on the other, hold promise of a readily performed clinical evaluation of pulmonary blood flow in cases of suspected intracardiac shunts, based on these two criteria.

The radiological appearances of pulmonary plethora were less frequently noted in our series of ventricular septal defects than was the case with the atrial septal defects. This may be evidence in support of Bedell's (1961) observation of a relatively lower pulmonary diffusing capacity in patients with ventricular septal defect. We were otherwise unable to confirm this assertion.

The effect of an increased pulmonary blood flow on the pulmonary diffusing capacity is not yet fully understood. The observed increase in diffusing capacity with exercise has been ascribed to the enhanced cardiac output. There is experimental evidence, however, that an increase in the cardiac output brought about in normal resting subjects is not accompanied by any significant changes in diffusing capacity (Ross, Frayser, and Hickam, 1959). This is to be expected when one considers the formula for $\mathrm{DL}_{\mathrm{co}}$ and its constituent factors (Roughton and Forster, 1957). The resistance to pulmonary $\mathrm{CO}$ diffusion $\left(1 / \mathrm{DL}_{\mathrm{CO}}\right)$ comprises two separate resistances, namely that which is offered by the alveolo-capillary membrane $\left(1 / \mathrm{D}_{\mathrm{M}}\right)$ and that which arises from the transfer of $\mathrm{CO}$ through the red cell membrane to the interior of the cell $\left(1 / \theta \mathrm{V}_{\mathrm{c}}\right)$. These two component resistances are arranged in series, and the total resistance may be expressed thus:

$$
\frac{1}{\mathrm{DL}_{\mathrm{CO}}}=\frac{1}{\mathrm{D}_{\mathrm{M}}}+\frac{1}{\theta \mathrm{V}_{\mathrm{C}}},
$$

where $D_{M}$ is the diffusing capacity of the alveolocapillary membrane, $V_{C}$ is the volume of blood in the pulmonary capillaries, and $\theta$ is a variable representing the rate of uptake of carbon monoxide by the red cells for each $\mathrm{mm}$. $\mathrm{Hg}$ carbon monoxide tension. It is apparent that the pulmonary blood flow, as such, does not, at least in theory, contribute to diffusion, but changes in the size of the pulmonary vascular bed do bear a direct relation to diffusing capacity. The rate of flow past the alveolocapillary membrane is not a critical factor, and the hæmoglobin "compartments" for carbon monoxide are so large as to preclude their speedy saturation (Bedell, 1961). An increased cardiac output flowing through a normal-sized capillary bed will thus not occasion an enhanced diffusing capacity. An enlarged capillary bed will, on the other hand, be associated with a greater rate of diffusion where 
the pulmonary flow too is increased. The existence of an increased pulmonary capillary blood volume, and consequently a greater capillary surface area available for diffusion, has been demonstrated in cases of intracardiac shunts by Rankin and Callies (1958). When these considerations are taken into account it is not to be expected that any sudden changes in pulmonary diffusing capacity will be effected consequent to the abrupt diminution in pulmonary flow, which must accompany closure of a septal defect. As has already been stated, this was in fact the experience in the current series of cases followed up after operation. We do not have at our disposal, however, any information regarding the possible shrinkage of total capillary volume in such lungs following corrective cardiac surgery.

A further point arising out of these results requires elucidation, namely the negative correlation which was found to exist between fractional $\mathrm{CO}$ uptake and pulmonary flow index. If this finding is significant, it seems to indicate that a critical point may well be reached in the rate of blood flow past the alveolo-capillary membrane. At this juncture, the increase in capillary surface area available for diffusion would, as it were, not be able to keep pace with the greatly accelerated blood flow, occasioning a relative inefficiency in alveolo-capillary gas transfer. The disproportionate interrelation arising from the logarithmic increase in blood volume over and against the linear increase in surface area available for diffusion will be evinced once the hypothetical critical point is attained. Recent work (Staub, 1963) has shown that the red cells normally do not, as was previously held to be the case, traverse the alveolar capillaries in single file. It seems reasonable to assume therefore that not all the circulating erythrocytes will take part in the diffusion process, and that relatively fewer cells will in fact be actively involved when peripheral capillary dilatation arises.

It seems that further studies, involving the direct measurement of the pulmonary capillary blood volume in patients with intracardiac shunts, would be valuable. Using a technique such as that of Roughton and Forster (1957), the relative participation of the component resistances to diffusion may be assessed. The factor $1 / \theta \mathrm{V}_{\mathrm{C}}$ is the more important of these, accounting on the average for some 50-70 per cent of the total resistance to diffusion, as against the $30-50$ per cent contribution of $1 / D_{M}$. In studying the pulmonary diffusing capacity of patients with intracardiac shunts uncomplicated by severe pulmonary hypertension and organic structural changes in the capillary membrane, the constituent resistance $1 / D_{M}$ may to a great extent be disregarded.

\section{SUMMARY}

An attempt has been made to devise a simple clinical test that would provide a reasonably accurate assessment of pulmonary blood flow in cases of suspected intracardiac shunts. In this connexion, the possibility of employing pulmonary diffusing capacity for carbon monoxide and the radiological appearances of the lung fields in routine chest radiographs as a guide to the grade of pulmonary flow has been evaluated.

Making use of an office procedure to measure the diffusion parameters of fractional $\mathrm{CO}$ uptake and CO "conductance", a series of 16 patients (with intracardiac shunts) who had previously undergone hæmodynamic studies and radiological assessment was compared with a group of normal subjects. A significantly higher $\mathrm{CO}$ uptake was found in the patients with intracardiac septal defects. There was no statistical difference between the mean values for $\mathrm{CO}$ conductance in the two groups. This was considered to indicate the absence of any over-all aberration in alveolo-capillary gas transfer in uncomplicated intracardiac shunts. There is some evidence, however, that both diffusion parameters may reflect a decreased diffusing capacity in cases of shunts complicated by pulmonary hypertension and membrane thickening.

No correlation was found to exist between either diffusion parameter and percentage left-to-right shunt, but a negative correlation has been demonstrated between CO uptake and pulmonary index. This can be explained in terms of a relative inefficiency in gas transfer resulting from the enhanced pulmonary flow.

No material changes in diffusion have been observed to attend closure of intracardiac septal defects. This seems to underline the importance of pulmonary capillary blood volume rather than pulmonary flow as a prime determinant of diffusing capacity.

It is concluded that carbon monoxide diffusion is a useful guide to the pulmonary circulation in cases of intracardiac shunts, and that a method of determining diffusing capacity that takes into account the size of the capillary bed and the surface area available for diffusion holds promise of an accurate assessment of pulmonary hæmodynamics in clinical application.

The authors wish to acknowledge the assistance of their colleagues Drs. I. van der Merwe, J. A. Beyers, and J. M. M. Basson, with the interpretation of chest radiographs, and Dr. R. L. M. Kotzé, Medical Superintendent, Karl Bremer Hospital, for permission to publish this report. 


\section{REFERENCES}

Bates, D. V. (1952). The uptake of carbon monoxide in health and in emphysema. Clin. Sci., 11, 21.

Bedell, G. N. (1961). Comparison of pulmonary diffusing capacity in normal subjects and in patients with intracardiac septal defects. F. Lab. clin. Med., 57, 269.

—, and Adams, R. W. (1962). Pulmonary diffusing capacity during rest and exercise. A study of normal persons and persons with atrial septal defect, pregnancy, and pulmonary disease. f. clin. Invest., 41, 1908.

Campbell, M. (1951). Visible pulsation in relation to blood flow and pressure in the pulmonary artery. Brit. Heart F., 13, 438.

Dornhorst, A. C. (1952). The assessment of respiratory function: an analytical review. St. Thom. Rep., 8 (2 Ser.), 69.

Fouché, R. F., Beck, W., and Schrire, V. (1963). The roentgenologic assessment of the degree of left-to-right shunt in secundum type atrial septal defect. Amer. $\mathcal{F}$. Roentgenol., 89, 254.

Healey, R. F., Dow, J. W., Sosman, M. C., and Dexter, L. (1949). The relationship of roentgenographic appearance of the pulmonary artery to pulmonary hemodynamics. Amer. F. Roentgenol., 62, 777.

Kraemer, W. F., Gensini, G., Blount, S. G., Jr., and Lanier, R. R. (1955). Roentgen aspects of atrial septal defect, ostium secundum. Acta radiol. (Stockh.), 44, 441.
MacNamara, J., Prime, F. J., and Sinclair, J. D. (1959). An assessment of the steady-state carbon monoxide method of estimating pulmonary diffusing capacity. Thorax, $14,166$.

Milledge, J. S. (1960). Carbon-monoxide diffusion: simple test for routine clinical use. Lancet, 1, 1051.

Rankin, J. (1960). Evaluation of alveolar capillary diffusion. In Clinical Cardiopulmonary Physiology, 2nd ed., pp. 624-634. Grune and Stratton, New York.

—, and Callies, Q. C. (1958). Diffusion characteristics of the human lung in congenital and acquired heart disease. Circulation, 18, 768. (Abstract.)

Ross, J. C., Frayser, R., and Hickam, J. B. (1959). A study of the mechanism by which exercise increases the pulmonary diffusing capacity for carbon monoxide. $\mathcal{F}$. clin. Invest., 38, 916.

Roughton, F. J. W., and Forster, R. E. (1957). Relative importance of diffusion and chemical reaction rates in determining rate of exchange of gases in the human lung, with special reference to true diffusing capacity of pulmonary membrane and volume of blood in the lung capillaries. F. appl. Physiol., 11, 290.

Staub, N. C. (1963). The interdependence of pulmonary structure and function. Anesthesiology, 24, 831.

Wassermann, H. P. (1961). Carbon monoxide diffusion. Experience with, and modification of an office procedure. S. Afr. med. F., 35, 598. 